\title{
MS35-P31 | DESIGN OF COCRYSTALS BASED ON ESSENTIAL OILS WITH A PALETTE OF
}

\section{DIFFERENT PROPERTIES}

Bacchi, Alessia (University of Parma, Parma, ITA); Carraro, Claudia (University of Parma, Parma, ITA); Mazzeo, Paolo Pio (University of Parma, Parma, ITA); Pelagatti, Paolo (University of Parma, Parma, ITA)

Cocrystals are multicomponent crystalline materials made by different chemical entities in stoichiometric ratio: designing a cocrystal requires a thorough knowledge of the possible interactions between the molecular partners. We present cocrystals containing liquid natural essential oils (EO), designed to tune the oil release profile at different environmental conditions. Appropriate supramolecular synthons between the EO molecule and suitable coformers provide a stable intermolecular network for the EO and increase the melting point stabilizing the liquid ingredient in a solid form. These supramolecular interactions also determine many fundamental physical properties of the material, e.g. solubility, hygroscopicity, thermal stability, density and mechanical strength. Thus cocrystallization offers great opportunities to create a palette of materials with empowered characteristics for processing, performance and shelf-life. We present the design of a library of cocrystals combining eugenol (extracted from clove buds and leaves), carvacrol (extracted from oregano) and thymol (the main component of thyme) with phenazine and hexamethylenetetramine to scan the range of properties that can be varied by pairing each EO with a different coformer. Coformers have been selected as rigid molecules acting as $\mathrm{H}$-bond acceptors. The six EO/coformer combinations resulted in six prototype cocrystals, which were fully characterized in terms of physical properties and in-vitro biological activities and compared with the pure EO. We show that these cocrystals span a broad range of properties, thus extending the applicability of these materials in various circumstances which might need different responses to external conditions, such as high or low ambient temperature, short term or long term release. 